\title{
Determinação da Fixação Biológica de Nitrogênio no Amendoim Forrageiro (Arachis spp.) por Intermédio da Abundância Natural de ${ }^{15} \mathrm{~N}$
}

\author{
Cesar Heraclides Behling Miranda ${ }^{1}$, Antonio Vieira ${ }^{2}$, Georg Cadisch ${ }^{3}$
}

\begin{abstract}
RESUMO - Quantificou-se a fixação biológica de nitrogênio (FBN) em cinco acessos de Arachis pintoi (BRA31534, BRA31828, BRA31796, BRA15121 e BRA30333) e dois de A. repens (BRA31801 e BRA31861). Os mesmos foram estabelecidos em um solo Latosolo Vermelho Escuro sujeito a inundação estacional, sendo a FBN estimada segundo a técnica da abundância natural do isótopo ${ }^{15} \mathrm{~N}\left(\mathrm{~d}^{15} \mathrm{~N}\right)$. Estolões dos acessos foram plantados em novembro de 1999, em parcelas de 2,0 m x 2,0 m, com quatro repetições, distribuídas em blocos ao acaso. A massa verde das plantas acima de cinco centímetros do solo foi colhida em janeiro de 2000 e seca em estufa a $65^{\circ} \mathrm{C}$ até peso constante, sendo posteriormente pesada e moída para análise dos conteúdos em $\mathrm{N}$ e d ${ }^{15} \mathrm{~N}$, em espectrômetro de massa. Verificaram-se diferenças significativas entre os genótipos quanto à produção de matéria seca (MS) e N total, sobressaindo-se BRA31534 e BRA31828, com produções de 4,2 t/ha e conteúdos totais de $\mathrm{N}$ de 102 e $110 \mathrm{~kg} / \mathrm{ha}$, respectivamente. Os acessos BRA30333 e BRA31861 produziram apenas 2,6 t de MS/ha, com 59 e $65 \mathrm{~kg} / \mathrm{ha}$ de $\mathrm{N}$ total, respectivamente. As taxas de FBN dos acessos testados, medidas por comparação dos seus teores de $\mathrm{d}^{15} \mathrm{~N}$ com os de plantas não fixadoras crescendo na mesma área, variaram de 36\% (BRA15121) a 90\% (BRA31828) do $\mathrm{N}$ total das plantas, equivalente a 26 e $99 \mathrm{~kg}$ de N/ha, respectivamente. Verificou-se correlação positiva e significativa $(\mathrm{r}=0,92, \mathrm{p}<0,05)$ entre os conteúdos totais de $\mathrm{N}$ da planta e os conteúdos totais de $\mathrm{N}$ obtidos de fixação biológica (NDF), e correlação negativa e significativa $(r=-0,93, p<0,01)$ entre NDF e o $\mathrm{N}$ total obtido do solo. Concluiu-se que a produção dos acessos mais promissores foi resultado de simbiose mais eficiente com as estirpes de Bradyrhizobium nativas do solo, com a FBN suprindo as necessidades nutricionais de $\mathrm{N}$ das plantas.
\end{abstract}

Palavras-chave: Bradyrhizobium, cerrados, leguminosas forrageiras, isótopos de ${ }^{15} \mathrm{~N}$, leguminosas

\section{Determination of Biological Nitrogen Fixation by the Forage Groundnut (Arachis spp.) Using the ${ }^{15} \mathrm{~N}$ Natural Abundance Technique}

\begin{abstract}
The biological nitrogen fixation (BNF) of five Arachis pintoi (BRA31534, BRA31828, BRA31796, BRA15121 E BRA30333) and two A. repens (BRA31801 e BRA31861) accessions, grown in a Dark Red Latosol prone to seasonal flooding was evaluated using the ${ }^{15} \mathrm{~N}$ natural abundance method $\left(\mathrm{d}^{15} \mathrm{~N}\right)$. Stolons of each accession were planted in November 1999 , in plots of 2.0 $\mathrm{m}$ by $2.0 \mathrm{~m}$, with four replications allotted to randomized blocks. Plant mass above five cm was harvested in January 2000 . There were significant differences among the tested accessions for dry matter (DM) production and total N content, with BRA31534 and BRA31828 producing $4.2 \mathrm{t} / \mathrm{ha}$ of DM, and 102 and $110 \mathrm{~kg} \mathrm{~N} / \mathrm{ha}$ respectively. BRA3033 and BRA31861 produced only $2.6 \mathrm{t} \mathrm{DM/ha}$ and 59 and $65 \mathrm{~kg} /$ ha of total $\mathrm{N}$, respectively. The proportion of $\mathrm{N}$ derived from $\mathrm{N}_{2}$ fixation, estimated by comparison of the $\mathrm{d}^{15} \mathrm{~N}$ of Arachis accessions with non $\mathrm{N}$-fixing plants growing in the same area, ranged from 36\% (BRA15121) to 90\% (BRA31128), equivalent to 26 and $99 \mathrm{~kg} \mathrm{~N} / \mathrm{ha}$, respectively. There was a positive and significant correlation $(\mathrm{r}=0.92, \mathrm{p}<0.05)$ between plant total $\mathrm{N}$ content and $\mathrm{N}$ derived from BNF, but a negative and significant correlation $(\mathrm{r}=-0.93, \mathrm{p}<0.001)$ was found between plant total $\mathrm{N}$ and $\mathrm{N}$ derived from soil. It was concluded that plant production by the most promising accessions was the result of an efficient symbiosis with soil Bradyrhizobium native strains, with BNF supplying plant $\mathrm{N}$ nutritional requirements.
\end{abstract}

Key Words: Bradyrhizobium, Brazilian savanna, forage legumes, ${ }^{15} \mathrm{~N}$ isotopes

\section{Introdução}

Entre as leguminosas do gênero Arachis citam-se algumas espécies forrageiras promissoras, tais como A. pintoi, A. repens e A. glabrata (Gregory et al., 1980; Grof, 1985; Kerridge \& Hardy, 1994). Com origem na região central da América do Sul, notadamente nos Cerrados e nas regiões costeiras do Brasil (Valls, 1992), o A. pintoi é uma leguminosa perene, de hábito de crescimento prostrado e com diversos estolões, o que dá origem a muitos pontos de crescimento, conferindo-lhe alta resistência à desfolha

\footnotetext{
1 Engo-Agro, PhD, Pesquisador Embrapa Gado de Corte. CP 154, CEP: 79002-970, Campo Grande, MS. Correio eletrônico: miranda@cnpgc.embrapa.br

2 Engo-Agro, Pesquisador Embrapa Gado de Corte. CP 154, CEP: 79002-970, Campo Grande, MS. Correio eletrônico: vieira@cnpgc.embrapa.br

${ }^{3}$ Department of Agricultural Sciences, Imperial College at Wye, Univ. of London. Wye, Ashford, Kent TN25 5AㅡZ, UK. E.mail: g.cadisch@ic.ac.uk
} 
pelo pastejo. Com isso, essa leguminosa cresce bem em associação com gramíneas agressivas tais como Brachiaria spp. (Lascano \& Thomas, 1988). De modo geral, as plantas do gênero Arachis são usadas comumente para cobertura do solo em fruticultura (Firth \& Wilson, 1995) ou para consorciação com gramíneas forrageiras nos trópicos úmidos (Bouman et al., 1999; Ibrahim \& t' Mannetje, 1998).

Além de serem tolerantes à alta saturação de alumínio e acidez do solo, as plantas do gênero Arachis têm como característica interessante a adaptação a solos com má drenagem, que podem ficar inundados por algum período do ano (Fernandes et al., 1992; Pizarro et al., 1992). Nos Cerrados, a maior região produtora de bovinos no País, (ANUALPEC, 2002), estima-se que existam cerca de 12 milhões de hectares nesta situação (Pizarro et al., 1992), com ocorrência de inundações durante o período das águas. Essas áreas não são melhor exploradas, devido ao desconhecimento de novas espécies forrageiras adaptadas a esses locais e de formas adequadas de manejo das pastagens nativas adaptadas a estas condições, as quais, em paralelo, apresentam baixa produção de forragem. Desse modo, a introdução de novas forrageiras, como plantas do gênero Arachis, nestas áreas, pode ser benéfica para o sistema produtivo.

A contribuição maior de Arachis ao sistema de produção, como de qualquer outra leguminosa, é seu potencial em fixar o nitrogênio $(\mathrm{N})$ atmosférico, quando em associação com bactérias fixadoras do gênero Bradyrhizobium, disso resultando uma forragem de melhor valor nutritivo, além da melhoria da fertilidade do solo. Por meio de simulação, estimouse que as leguminosas forrageiras podem fazer com que o balanço de $\mathrm{N}$ em áreas de pastagens seja positivo (Cadisch et al., 1994; Thomas, 1995).

Em um estudo com Arachis pintoi CIAT 17434 em dois solos da Colômbia, usando-se a diluição isotópica do ${ }^{15} \mathrm{~N}$ como forma de estimar as taxas de fixação de $\mathrm{N}_{2}$, observou-se que esta espécie pode fixar até $80 \%$ de suas demandas nutricionais em N. A mesma taxa de fixação foi medida em Centrosema acutifolium e Stylosanthes capitata crescendo nas mesmas condições (Thomas et al., 1997). Os autores concluíram, então, que estas taxas podem ser assumidas como de ocorrência comum em leguminosas forrageiras consorciadas com gramíneas tropicais, quando estas estiverem crescendo em condições de disponibilidade limitada de $\mathrm{N}$ do solo, porém sem limitações de outras necessidades básicas de crescimento (nutrição e condições edafo-climáticas adequadas).

No presente estudo foram mensurados as taxas de fixação de $\mathrm{N}_{2}$ de cinco acessos de A. pintoi e de dois acessos de $A$. repens crescendo em monocultura em um solo que sofre inundação, na estação das águas, em área dos Cerrados de Campo Grande, MS.

Para a determinação da fixação de $\mathrm{N}_{2}$ utilizou-se a técnica da abundância natural do isótopo ${ }^{15} \mathrm{~N}$. Esta técnica toma por base o fato de que o $\mathrm{N}$ mineral do solo é normalmente um pouco enriquecido com ${ }^{15} \mathrm{~N}$, como resultado de fracionamento isotópico entre ${ }^{14} \mathrm{~N}$ e ${ }^{15} \mathrm{~N}$ que ocorre nos processos físicos, químicos e biológicos que envolvem o $\mathrm{N}$ da matéria orgânica e do solo (Shearer \& Kohl, 1986). Dessa maneira, uma planta não fixadora do $\mathrm{N}_{2}$ da atmosfera, crescendo nessas condições, terá sua composição em ${ }^{15} \mathrm{~N}$ semelhante a do $\mathrm{N}$ disponível do solo. Por outro lado, uma planta fixadora do $\mathrm{N}_{2}$ da atmosfera apresentará teores menores de ${ }^{15} \mathrm{~N}$, devido ao efeito de diluição que esse $\mathrm{N}_{2}$ causará, uma vez que o ${ }^{15} \mathrm{~N}$ em excesso da atmosfera é zero. Assim, usando-se uma planta não fixadora como marcadora do ${ }^{15} \mathrm{~N}$ do $\mathrm{N}$ mineral do solo, a taxa de fixação pode ser determinada pela proporção com que este ${ }^{15} \mathrm{~N}$ foi diluído (Shearer \& Kohl 1986). Na prática, é impossível saber se uma dada planta não obtém algum $\mathrm{N}$ oriundo de fixação, por mecanismos associados ou não à planta. Além disso, idealmente, uma planta não fixadora deveria explorar um volume de solo semelhante ao da fixadora, apresentando, ainda, padrões de absorção e assimilação do $\mathrm{N}$ parecidos. Como isso é praticamente impossível de ser atendido, usa-se a média do ${ }^{15} \mathrm{~N}$ de algumas plantas reconhecidamente de espécies para as quais não há histórico comprovado de fixação biológica associada que estão crescendo nas mesmas condições das fixadoras. Um exemplo prático da aplicação dessa técnica em leguminosas tropicais é apresentado por Miranda et al. (1999).

\section{Material e Métodos}

O experimento foi estabelecido em um Latosolo Vermelho Escuro com cobertura vegetal natural tipo Campo Cerrado, com impedimento de drenagem causada por uma camada endurecida de plintita a cerca de $40 \mathrm{~cm}$ de profundidade. O solo é constituído de $58 \%$ de areia, $33 \%$ de argila e $8 \%$ de silte. A análise 
química do solo indicou pH em água igual a 4,3; teor de matéria orgânica de 2,9\%; teor de P (Mehlich-1) menor do que $1 \mathrm{mg} / \mathrm{g}$ e teores de $\mathrm{Ke} \mathrm{Ca}+\mathrm{Mg}$ de 0,05 e $2,0 \mathrm{cmol}_{\mathrm{c}} / \mathrm{kg}$, respectivamente. Antes do plantio a área foi preparada mecanicamente (uma passagem de arado de aiveca e uma gradagem leve), corrigida com $2 \mathrm{t} /$ ha de calcário dolomítico e fertilizada com o equivalente a $200 \mathrm{~kg} / \mathrm{ha}$ de $\mathrm{P}_{2} \mathrm{O}_{5}$ (superfosfato simples), $150 \mathrm{~kg} / \mathrm{ha}$ de $\mathrm{K}_{2} \mathrm{O}$ (cloreto de potássio) e $40 \mathrm{~kg} / \mathrm{ha}$ de fritas (FTE BR10). O corretivo e adubação foram incorporados por uma nova gradagem leve.

Em dezembro de 1998, estolões de sete acessos de A. pintoi (BRA15121, BRA22683, BRA30333, BRA30872, BRA31496, BRA31534, e BRA31828), dois de A. repens (BRA31801 e BRA31861) e a variedade de $A$. pintoi CIAT 17434 (registrada no Brasil como BRA13251 e na Austrália como cv. Amarillo) foram plantados em parcelas de $2,0 \mathrm{~m} \times 2,0 \mathrm{~m}$, com quatro repetições, em um desenho experimental de blocos ao acaso. Utilizaram-se 10 covas por parcela.

Até abril de 1999 a área experimental esteve alagada, especialmente em janeiro e março, observando-se constantemente uma pequena lâmina de água superficial, uma vez que a área é plana, sem escorrimento superficial, complementado pelo impedimento de drenagem causado pela camada de plintita na sub-superfície. Porém, de maio a outubro, ocorreu uma seca severa. Com isso, os acessos CIAT17434, BRA22683, BRA30872 e BRA31496 não se estabeleceram apropriadamente, cobrindo somente $10 \%$ da área da parcela até a ocasião da colheita. Em janeiro de 2000, uma área interna da parcela, de $50 \mathrm{~cm}$ x $50 \mathrm{~cm}$, foi colhida nas quatro repetições dos acessos que estavam totalmente estabelecidos, ou seja, que tinham a área da parcela totalmente coberta. As plantas foram cortadas a $5 \mathrm{~cm}$ do solo e as amostras foram secas a $65^{\circ} \mathrm{C}$ por 72 horas. Após a secagem, as amostras foram pesadas, moídas finamente e armazenadas para análises posteriores. Em paralelo, amostras de quatro parcelas de Panicum maximum cv. Massai, e das plantas invasoras Solanum viarum, Sonchus spp., Sida rhombifolia e Panicum repens, que estavam crescendo na mesma área, sob as mesmas condições de adubação, foram colhidas e processadas da mesma maneira. Estas plantas foram usadas como plantas controles não fixadoras de $\mathrm{N}_{2}$, isto é, como marcadores do $\mathrm{d}^{15} \mathrm{~N}$ do $\mathrm{N}$ mineral do solo. Finalmente, as amostras foram analisadas diretamente quanto aos seus conteúdos de $\mathrm{N}$ total e ${ }^{15} \mathrm{~N}$ usando-se um auto-analisador de Ce N (ROBOPREP $\mathrm{CN}$ ) acoplado a um espectrômetro de massa modelo SL20-20 (Europa Scientific, Crewe, UK).

Os cálculos das taxas de fixação de $\mathrm{N}_{2}$ foram feitos usando-se a equação proposta por AMARGER et al. (1979). De forma geral, tomam-se as diferenças entre o $\mathrm{d}^{15} \mathrm{~N}$ da planta fixadora e $o \mathrm{~d}^{15} \mathrm{~N}$ da planta controle não fixadora como proporções do $\mathrm{d}^{15} \mathrm{~N}$ da planta controle não fixadora. $\mathrm{O} \mathrm{d}{ }^{15} \mathrm{~N}$ é definido como $\mathrm{d}^{15} \mathrm{~N}(\% o)=\left[\left({ }^{15} \mathrm{~N} /{ }^{14} \mathrm{~N}\right.\right.$ amostra $/{ }^{15} \mathrm{~N} /{ }^{14} \mathrm{~N}$ padrão $)$ - 1] x 1000, onde o padrão é o $\mathrm{N}_{2}$ atmosférico, cuja abundância de ${ }^{15} \mathrm{~N}$ é de $0,3663 \%$. Como ocorrem algumas discriminações físicas entre os isótopos durante o processo de redução do $\mathrm{N}_{2}$, um fator de correção, chamado b, é introduzido. A rigor, é o d ${ }^{15} \mathrm{~N}$ que a planta fixadora tem quando em completa dependência de fixação. Um valor b de $0,07 \mathrm{~d}^{15} \mathrm{~N}$ foi usado a partir da média mostrada por quatro acessos de A. pintoi (CIAT 14343, 48744, 18748, e 22160) crescendo em condições controladas e inoculados com a estirpe de Bradyrhizobium spp. CIAT 3101 (Mora, 2001).

\section{Resultados e Discussão}

Verificaram-se diferenças significativas $(\mathrm{p}<0,01)$ na massa seca e nos conteúdos de $\mathrm{N}$ total dos diferentes acessos testados (Tabela 1), o que indica graus diferentes de adaptação às condições locais. $\mathrm{O}$ período de inundação após o plantio, de janeiro a março, seguido de uma seca severa, de maio a outubro, contribuiu negativamente para o estabelecimento de alguns acessos, inclusive da variedade padrão (CIAT 17434). Entretanto, outros acessos estabeleceram-se, apesar dessas condições adversas, o que enfatiza a necessidade de mais estudos sobre a adaptabilidade de novos materiais em ambientes específicos, especialmente considerando que estes acessos foram selecionados a partir de uma coleção inicial testada em outros locais.

Os acessos BRA31534 e BRA31828 produziram matéria seca acima de 4 t/ha, o que pode ser considerado boa produção para o primeiro ano, uma vez que esta espécie apresenta estabelecimento lento. Essas quantidades são similares ao que Pizarro et al. (1992) encontraram em A. pintoi crescendo também em uma área de baixada em outra região dos Cerrados. Os acessos BRA31801 e BRA31796 também mostraram boa produção, cerca de $3,8 \mathrm{t} / \mathrm{ha}$, mas o BRA30333 e o BRA31861, com produção de somente 
Tabela 1 - Massa seca (t/ha), teores de N (\%) e $\mathrm{N}$ total ( $\mathrm{kg} / \mathrm{ha}$ ) de sete acessos de Arachis spp. no primeiro ano de crescimento em um Latosolo Vermelho Escuro. Cada valor é média de quatro repetições

Table 1 - Dry weight (t/ha), N content (\%) and total N ( $\mathrm{kg} / \mathrm{ha}$ ) of seven Arachis spp. accessions in the first year of growth in a Dark Red Latossol. Each value is the mean of four replications

\begin{tabular}{llccc}
\hline $\begin{array}{l}\text { Espécies } \\
\text { Species }\end{array}$ & $\begin{array}{c}\text { Acessos } \\
\text { Accession }\end{array}$ & $\begin{array}{c}\text { Massa seca } \\
\text { Dryweight } \\
(\mathrm{t} / \mathrm{ha})\end{array}$ & $\mathrm{N}$ & $\begin{array}{l}\mathrm{N} \text { total } \\
\text { Total N } \\
(\mathrm{kg} / \mathrm{ha})\end{array}$ \\
\hline A. pintoi & BRA31534 & $4,2^{\mathrm{a}}$ & 2,39 & $102^{\mathrm{ab}}$ \\
A. pintoi & BRA31828 & $4,2^{\mathrm{a}}$ & 2,63 & $110^{\mathrm{a}}$ \\
A. repens & BRA31801 & $3,9^{\mathrm{ab}}$ & 2,60 & $101^{\mathrm{ab}}$ \\
A. pintoi & BRA31796 & $3,8^{\mathrm{abc}}$ & 2,59 & $97^{\mathrm{abc}}$ \\
A. pintoi & BRA 15121 & $3,3^{\mathrm{abc}}$ & 2,13 & $69^{\mathrm{bcd}}$ \\
A. pintoi & BRA30333 & $2,7^{\mathrm{bc}}$ & 2,22 & $59^{\mathrm{d}}$ \\
A. repens & BRA31861 & $2,6^{\mathrm{c}}$ & 2,47 & $65^{\mathrm{cd}}$ \\
\hline
\end{tabular}

Valores seguidos de mesma letra na coluna não diferem entre si (Duncan, $p<0,05$ ).

Values followed by the same letter in the column do not differ (Duncan, $p<.05$ ).

2,6 t/ha de matéria seca, não se mostraram promissores para o sistema produtivo da área testada.

Os teores percentuais de $\mathrm{N}$ dos acessos testados (Tabela 1) variaram de 2,13\% no BRA15121 a 2,63\% no BRA31828. Já o conteúdo de $\mathrm{N}$ total dos acessos variou de $59 \mathrm{~kg} / \mathrm{ha}($ BRA30333) a $110 \mathrm{~kg} / \mathrm{ha}$ (BRA31828).

Os acessos de A. pintoi e A. repens mostraram depleção significativa $(\mathrm{p}<0,01)$ de $\mathrm{d}^{15} \mathrm{~N}$ em comparação às plantas controle, não fixadoras de $\mathrm{N}_{2}$ da atmosfera (Tabela 2). Os acessos BRA30333 e BRA15121 mostraram os maiores valores de $\mathrm{d}^{15} \mathrm{~N}$, enquanto os demais acessos mostraram valores mais baixos e muito próximos entre si. Já as plantas controles não fixadoras, incluindo o $P$. maximum cv. Massai, mostraram valores de $\mathrm{d}^{15} \mathrm{~N}$ similares, com média $6,99 \delta^{15} \mathrm{~N}$. Isto sugere que o $\mathrm{N}$ mineral do solo teve, aparentemente, um enriquecimento em $\mathrm{d}^{15} \mathrm{~N}$ estável, o que aumenta o grau de confiança de aplicabilidade da técnica da abundância natural deste isótopo. Também possibilita inferir que a escolha dessas plantas como referência não deve ter introduzido grandes erros na avaliação das taxas de fixação. A escolha de plantas de referência é um parâmetro muito discutível na técnica de diluição isotópica do ${ }^{15} \mathrm{~N}$, conforme abordado por Chalk \& Ladha (1999). O uso de várias plantas de referência foi proposto por Boddey et al. (1995), enquanto Pate et al. (1994) sugeriram o uso de amostragem pareada (amostras crescendo o mais próximo possível, porém sem competição entre si) para minimizar possíveis erros devidos a variações espaciais. Estas recomendações foram atendidas no presente trabalho.

Considerando que a média dos valores de $\mathrm{d}^{15} \mathrm{~N}$ das plantas controle não fixadoras representa a média do $\mathrm{d}^{15} \mathrm{~N}$ do $\mathrm{N}$ mineral do solo disponível no período, calcularam-se as taxas de fixação de cada acesso, que variaram de $36 \%$ (BRA15121) a $90 \%$ (BRA31828) (Tabela 2). Nos acessos mais promissores (BRA31534, BRA31828, BRA31801 e BRA31796), constataram-se taxas acima de $74 \%$ e nos de menor produção, taxas menores (BRA30333 $=54 \%$ e BRA $15121=36 \%$ ). A única exceção foi constatada no acesso BRA31861, no qual se verificou alta taxa de fixação (88\%), apesar da baixa produção de matéria seca, indicando que outros mecanismos que não a disponibilidade de $\mathrm{N}$ são limitantes à produção desse acesso.

Essas diferentes taxas de fixação do $\mathrm{N}_{2}$ resultaram das diferentes habilidades dos acessos testados quanto ao estabelecimento da simbiose com estirpes nativas de Bradyrhizobium, uma vez que as plantas não foram inoculadas no plantio. Considerando que a área utilizada possuía muitas leguminosas nativas, admite-se que o solo tinha uma população de Bradyrhizobium estabelecida. Entretanto, Sá et al. (2001) encontraram baixa população de estirpes infectantes de A. pintoi em solos de Cerrados sem a presença desta leguminosa. Por outro lado, a inoculação com estirpes mais eficientes poderia ter sido benéfica para os acessos BRA15121 e BRA30333, uma vez que, aparentemente, não havia, para esses acessos, estirpes da bactéria ideais para maior expressão da interação simbiótica. Esta também pode ser uma das causas da falha de estabelecimento dos outros acessos testados. Mora (2001) também verificou que, em média, acessos de A. pintoi inoculados com a estirpe CIAT3101, de reconhecida eficiência, 
Tabela 2 - Abundância natural de ${ }^{15} \mathrm{~N}\left(\mathrm{~d}^{15} \mathrm{~N}\right)$, taxas de fixação de $\mathrm{N}_{2}$ (\%) e $\mathrm{N}$ derivado da fixação ou do solo (kg/ha) dos sete acessos de Arachis spp. Cada valor é média de quatro repetições

Table 2 - Natural abundance of ${ }^{15} \mathrm{~N}\left(\mathrm{~d}^{15} \mathrm{~N}\right)$, rates of $\mathrm{N}_{2}$ fixation (\%) and amount of $\mathrm{N}$ derived from fixation or from the soil (kg/ha) for seven accessions of Arachis spp. Each value is the mean of four replications

\begin{tabular}{|c|c|c|c|c|c|}
\hline $\begin{array}{l}\text { Espécies } \\
\text { Species }\end{array}$ & $\begin{array}{c}\text { Acessos } \\
\text { Accessions }\end{array}$ & $\delta^{15} \mathrm{~N}$ & $\begin{array}{c}\text { Taxas de } \\
\text { fixação de } \mathrm{N}_{2} \\
\text { Rates of } \\
N_{2} \text { fixation } \\
(\%)\end{array}$ & $\begin{array}{l}\mathrm{N} \text { derivado } \\
\text { de fixação } \\
N \text { derived } \\
\text { from fixation } \\
(\mathrm{kg} / \mathrm{ha})\end{array}$ & $\begin{array}{l}\mathrm{N} \text { derivado } \\
\text { do solo } \\
N \text { derived } \\
\text { from the soil }\end{array}$ \\
\hline A. pintoi & BRA 31534 & $1,14^{\mathrm{d}}$ & $85^{\mathrm{a}}$ & $85^{\mathrm{ab}}$ & $17^{\mathrm{bc}}$ \\
\hline A. pintoi & BRA 31828 & $0,73^{\mathrm{d}}$ & $90^{\mathrm{a}}$ & $99^{\mathrm{a}}$ & $11^{\mathrm{bc}}$ \\
\hline A. repens & BRA 31801 & $1,18^{\mathrm{d}}$ & $87^{\mathrm{a}}$ & $86^{\mathrm{ab}}$ & $15^{\mathrm{bc}}$ \\
\hline A. pintoi & BRA 31796 & $1,84^{\mathrm{d}}$ & $74^{\mathrm{a}}$ & $71^{\mathrm{ab}}$ & $26^{\mathrm{b}}$ \\
\hline A. pintoi & BRA 15121 & $4,53^{b}$ & $36^{\mathrm{b}}$ & $26^{\mathrm{d}}$ & $43^{\mathrm{a}}$ \\
\hline A. pintoi & BRA 30333 & $3,26^{\mathrm{c}}$ & $54^{\mathrm{b}}$ & $34^{\mathrm{cd}}$ & $25^{\mathrm{b}}$ \\
\hline A. repens & BRA 31861 & $1,14^{\mathrm{d}}$ & $88^{\mathrm{a}}$ & $57^{\mathrm{cd}}$ & $8^{c}$ \\
\hline Média dos controles & & $6,99^{\mathrm{a}}$ & - & - & - \\
\hline \multicolumn{6}{|l|}{ Control average } \\
\hline Solanum viarum & & 7,28 & & & \\
\hline Sonchus spp. & & 6,75 & & & \\
\hline Sida rhombifolia & & 6,79 & & & \\
\hline Panicum repens & & 6,40 & & & \\
\hline P. maximum & & 7,67 & & & \\
\hline
\end{tabular}

Valores seguidos de mesma letra na coluna não diferem entre si (Duncan, $p<0,05)$.

Values followed by the same letter in the column do not differ (Duncan, $p<.05$ ).

produziram mais e tiveram taxas mais altas de fixação de $\mathrm{N}_{2}$ comparativamente a quando em simbiose com estirpes nativas de um solo do México. Entretanto, o autor mostrou que os acessos de A. pintoi tiveram resposta diferenciada entre si quando em simbiose com as estirpes nativas.

Deve-se considerar que estas taxas de fixação foram obtidas com as plantas crescendo somente em competição com seus pares, por estarem em parcelas isoladas. Se elas estivessem crescendo em competição com outras espécies, como quando em consorciação com gramíneas, outros fatores competitivos entrariam em ação. Numa situação dessas, se as leguminosas não estivessem obtendo pelo menos $80 \%$ das suas necessidades em $\mathrm{N}$ através de fixação, como descrito por Thomas et al. (1997), teriam poucas chances de sobrevivência por longo tempo.

Entre 26 (BRA15121) e $99 \mathrm{~kg}$ de N/ha (BRA31828) foram obtidos através de fixação de $\mathrm{N}_{2}$ pelos acessos testados (Tabela 2). Verificouse correlação positiva e significativa $(r=0,92$, $\mathrm{p}<0,05)$ entre o conteúdo de $\mathrm{N}$ total das plantas e o conteúdo de $\mathrm{N}$ derivado de fixação, embora a correlação entre o conteúdo de $\mathrm{N}$ total com as taxas de fixação tenha sido fraca e não significativa $(\mathrm{r}=0,64, \mathrm{p}>0,05)$ (Tabela 3). Por outro lado, as taxas de fixação de $\mathrm{N}_{2}$ correlacionaram-se negativa e significativamente com os conteúdos de $\mathrm{N}$ originado do solo $(r=-0,93, p<0,01)$.

Considerando o $\mathrm{N}$ das plantas originado do solo como um indicador do potencial do solo em fornecer $\mathrm{N}$, admite-se que pelo menos $40 \mathrm{~kg} / \mathrm{ha}$ de $\mathrm{N}$ estavam disponíveis no período em estudo. Esta foi a quantidade

Tabela 3 - Correlações entre $N$ total (NT), massa seca (MS), taxas de fixação de $\mathrm{N}_{2}$ (\%NF), $\mathrm{N}$ derivado de fixação (NDF) ou do solo (NDS) nos sete acessos de Arachis spp.

Table 3 - Correlation among total N (NT), dry weight (MS), rates of $\mathrm{N}_{2}$ fixation (\%NF), $N$ derived from fixation (NDF) or from the soil (NDS) by seven accessions of Arachis spp.

\begin{tabular}{lcc}
\hline & $\begin{array}{c}\text { Correlação } \\
\text { Correlation }\end{array}$ & $\begin{array}{c}\text { Nível de probabilidade } \\
\text { Probability }\end{array}$ \\
\hline NT x MS & 0,96 & 0,000 \\
NT x \% NF & 0,64 & 0,113 \\
NTx NDF & 0,92 & 0,002 \\
TN x NDS & $-0,37$ & 0,412 \\
\% NF x NDF & 0,89 & 0,005 \\
\% NFx NDS & $-0,93$ & 0,001 \\
\% NF x MS & 0,43 & 0,326 \\
NDF x NDS & $-0,71$ & 0,066 \\
NDF x MS & 0,78 & 0,029 \\
NDS x MS & $-0,14$ & 0,758 \\
\hline
\end{tabular}


máxima medida no acesso com a menor taxa de fixação (BRA15121, com 36\%) e a mais alta recuperação de $\mathrm{N}$ do solo (Tabela 2). A maioria dos outros acessos, entretanto, não usou este $\mathrm{N}$ disponível, utilizando, preferencialmente, $\mathrm{o} \mathrm{N}$ originado da fixação. Essa economia de $\mathrm{N}$ do solo pode ser benéfica para a gramínea porventura associada ou para outra cultura subseqüente, pelo chamado sparring effect, como demonstrado em estudo com a leguminosa grão-de-bico (Cicer arietinum) por Herridge et al. (1995). Os acessos BRA31534, BRA31828, BRA31801 e BRA31796 fizeram uso eficiente da simbiose e do $\mathrm{N}$ do solo (Tabela 2). O acesso BRA31861 não usou todo esse $\mathrm{N}$ disponível no solo, mostrando dependência do $\mathrm{N}$ derivado de fixação (88\% do seu $\mathrm{N}$ total). O acesso BRA30333 extraiu N do solo em quantidade semelhante aos acessos mais produtivos, mas teve sua produção de matéria seca um pouco comprometida por menor taxa de fixação (54\% do seu $\mathrm{N}$ total). Essa variação nos resultados sugere que algum outro fator, à parte do N, limitou a produção desses acessos. Cadisch et al. (1989) mostraram que a deficiência de $\mathrm{PK}$ pode reduzir significativamente a fixação biológica de leguminosas forrageiras. Entretanto, como o solo foi corrigido e fertilizado com fósforo, potássio e micronutrientes no plantio, não há razão para se pensar que deficiência desses nutrientes essenciais tenha sido a maior causa dessa baixa produção. Dessa forma, acredita-se que esse fato seja decorrente de baixo potencial de produção de matéria seca por unidade de $\mathrm{N}$ absorvido, que é corroborado pelos baixos percentuais de $\mathrm{N}$ na matéria seca dos acessos BRA30333 e BRA15121 (Tabela 1).

\section{Conclusões}

Verificaram-se diferenças no potencial produtivo e na dependência em fixação de $\mathrm{N}_{2}$ dos diferentes acessos de A. pintoi e A. repens, quando crescendo em um solo periodicamente inundável dos Cerrados.

A produção de massa seca das plantas foi bem correlacionada com o $\mathrm{N}$ das plantas derivado de fixação biológica de $\mathrm{N}_{2}$, o que indica eficiência da simbiose estabelecida com estirpes de Bradyrhizobium nativas do solo.

Recomenda-se ênfase na avaliação do potencial de fixação de $\mathrm{N}_{2}$ de leguminosas forrageiras quando do estudo de sua adaptação a ambientes diversos, além do seu potencial de massa seca de forragem.

\section{Literatura Citada}

AMARGER, N.; MARIOTTI, A.; MARIOTTI, F. et al. Estimate of symbiotically fixed nitrogen in field grown soybeans using variations in ${ }^{15} \mathrm{~N}$ natural abundance. Plant and Soil, v.52, n.2, p.269-280,1979.

ANUALPEC 2002. Anuário da Pecuária Brasileira. São Paulo: FNP Consultoria e Comércio/Argos, 2002. 400p.

BODDEY, R.M.; OLIVEIRA, O.C.; ALVES, B.J.R. et al. Field application of the ${ }^{15} \mathrm{~N}$ isotope dilution technique for the reliable quantification of plant-associated biological nitrogen fixation. Fertility Research, v.42, n.1-3, p.77-87, 1995.

BOUMAN, B.A.M.; NIEUWENHUYSE, A.; IBRAHIM, M. Pasture degradation and restoration by legumes in humid tropical Costa Rica. Tropical Grassland, v.33 n.2, p.98-110, 1999.

CADISCH, G.; SYLVESTER-BRADLEY, R.; NÖSBERGER, J. ${ }^{15} \mathrm{~N}$ based estimation of nitrogen fixation of eight tropical forage legumes at two levels of P:K supply. Field Crops Research, v.22, n.3, p.181-194, 1989.

CADISCH, G.; SCHUNKE, R.M.; GILLER, K.E. Nitrogen cycling in a pure grass pasture and a grass-legume mixture on a red Latossol in Brazil. Tropical Grassland, v.28, n.1, p.43-52, 1994.

CHALK, P.M.; LADHA, J.K Estimation of legume symbiotic dependence: an evaluation of techniques based on ${ }^{15} \mathrm{~N}$ dilution. Soil Biology and Biochemistry, v.31, n.14, p.1901-1917, 1999.

FERNANDES, A.T.F.; FERNANDES, C.D.; EUCLIDES, V.P.B. et al. Avaliação de acessos de Paspalum spp em consorciação com Arachis pintoi em áreas umidas de baixa fertilidade. In: RED INTERNACIONAL DE EVALUACION DE PASTOS TROPICALES, 1., 1992, Brasília. Reunión Sabanas... Cali: Embrapa-CPAC/CIAT, 1992. p.555-560./ (Documento de Trabajo, 117).

FIRTH, D.J.; WILSON, G.P.M. Preliminary evaluation of species for use as permanent ground cover in orchards on the North coast of New-South-Wales. Tropical Grassland, v.29, n.1, p.18-27, 1999.

GROF, B. Forage attributes of the perennial groundnut Arachis pintoi in the tropical savanna environment in Colombia. In: INTERNATIONAL GRASSLAND CONGRESS, 15., 1985, Kyoto. Proceedings... Nagoya: The Japanese Society of Grassland Science, 1985. p.168-170.

GREGORY, W.C.; KRAPOVICKAS, A.; GREGORY, M.P. Structure, variation, evolution and classification in Arachis. In: SUMERFIELD, R.J.; BUNTING, A.H. (Eds.) Advances in legume sciences. Kew: Kew Royal Botanic Gardens, 1980. p.469-481.

HERRIDGE, D.F., MARCELLOS, H., FELTON, W.L. et al. Chickpea increases soil-N fertility in cereal systems through nitrate sparring and $\mathrm{N}_{2}$ fixation. Soil Biology and Biochemistry, v.27, n.4, p.545-551, 1995.

IBRAHIM, M.A.; t'MANETJE, L. Compatibility, persistence and productivity of grass-legume mixtures in the humid tropics of Costa Rica. 1. Dry matter yield, nitrogen yield and botanical composition. Tropical Grassland, v.32, n.1, p.96-104, 1998.

KERRIDGE, P.C.; HARDY, B. Biology and agronomy of forage Arachis. Cali: CIAT, 1994. 171p.

LASCANO, C.E.; THOMAS, D. Forage quality and animal selection of Arachis pintoi in association with tropical grasses in the eastern plains of Colombia. Grass and Forage Science, 
v.43, p.433-439, 1988

MIRANDA, C.H.B.; FERNANDES, C.D.; CADISCH, G. Quantifying the nitrogen fixed by Stylosanthes spp. Pasturas Tropicales, v.21, p.64-69, 1999.

MORA, B.V. DE LA. Contribution of the forage legume Arachis pintoi to soil fertility in a tropical pasture system in Vera Cruz, Mexico. Londres: Department of Biology, Imperial College at Wye, University of London, 2001.210 p. Tese (Doutorado em Agricultural Sciences) - University of London, 2001.

PATE, J.S.; UNKOVICH, M.J.; ARMSTRONG, E.L. et al. Selection of reference plants for ${ }^{15} \mathrm{~N}$ natural abundance assessment of $\mathrm{N}_{2}$ fixation by crop and pasture legumes in southwest Australia. Australian Journal of Agricultural Research, v.45, n.1, p.133-47,1994.

PIZARRO, E.A.; CARVALHO, M.A.; VALLS, J.F.M. et al. Arachis spp: evaluacíon agronomica en areas bajas del Cerrado. In: RED INTERNACIONAL DE EVALUACION DE PASTOS TROPICALES, 1, 1992, Brasília. Reunión Sabanas... Cali: Embrapa-CPAC/CIAT, 1992. p.353-356. (Documento de Trabajo, 117)

SÁ, N.M.H.; PINTO, P.P.; PASSOS, R.V.M. et al. Seasonal dynamic of native rhizobial populations associated with Arachis pintoi in Cerrado soils. Pasturas Tropicales, v.23, n.1, p.29-31, 2001.
SHEARER, G.; KOHL, D.H. $\mathrm{N}_{2}$ fixation in field setting: estimations based on natural ${ }^{15} \mathrm{~N}$ abundance. Australian Journal of Plant Physiology, v.13, n.6, p.699-756, 1986.

THOMAS, R.J. Role of legumes in providing $\mathrm{N}$ for sustainable tropical pasture legumes. Plant and Soil, v.174, n.1-2, p.103-118, 1995.

THOMAS, R.J.; ASAKAWA, N.M.; RONDON, M.A. et al. Nitrogen fixation by three tropical forage legumes in an acidsoil savanna of Colombia. Soil Biology and Biochemistry, v.29, n.5-6, p.801-808, 1997.

VALLS, J.F.M. Origem do germoplasma de Arachis pintoi disponível no Brasil. In: RED INTERNACIONAL DE EVALUACION DE PASTOS TROPICALES, 1., 1992, Brasília. Reunión Sabanas... Cali: Embrapa-CPAC / CIAT, 1992. p.81-96. (Documento de Trabajo, 117)

Recebido em: 17/04/02 Aceito em: 12/05/03 